\title{
Insomnia as an independent predictor of suicide attempts: a nationwide population- based retrospective cohort study
}

Han-Ting Lin ${ }^{1}$, Chi-Huang Lai ${ }^{1}$, Huey-Jen Perng ${ }^{2}$, Chi-Hsiang Chung ${ }^{2}$, Chung-Ching Wang ${ }^{3}$, Wei-Liang Chen ${ }^{3}$ and Wu-Chien Chien ${ }^{1,2,4^{*}}$ (D)

\begin{abstract}
Background: Numerous studies have verified that insomnia is associated with suicidal ideation, suicide attempts, and death by suicide. Limited population-based cohort studies have been conducted to examine the association. The present study aimed to analyze whether insomnia increases the risk of suicide attempts and verify the effects of insomnia on suicide risk.

Methods: This study is a cohort study using 2000-2013 hospitalization data from the National Health Insurance Research Database (NHIRD) to track the rate of suicide attempts among insomnia patients aged 15 years or older. In addition, a 1:2 pairing based on sex, age, and date of hospitalization was conducted to identify the reference cohort (patients without insomnia). Cox proportional hazard model was used to assess the effects of insomnia on suicide risk.

Results: The total number of hospitalized patients aged 15 years or older was 479,967 between 2000 and 2013 (159,989 patients with insomnia and 319,978 patients without insomnia). After adjusting for confounders, suicide risk in insomnia patients was 3.533-fold that of patients without insomnia (adjusted hazard ratio [HR] $=3.533,95 \%$ confidence interval [Cl] $=3.059-4.080, P<0.001$ ). Suicide risk in low-income patients was 1.434-fold (adjusted $\mathrm{HR}=1.434,95 \% \mathrm{Cl}=1.184-1.736, P$ $<0.001$ ) that of non-low-income patients. Suicide risk in patients with drug dependence and with mental disorders was 1. 592-fold (adjusted $\mathrm{HR}=1.592,95 \% \mathrm{Cl}=1.220-2.077, P<0.001$ ) and 4.483-fold (adjusted $\mathrm{HR}=4.483,95 \% \mathrm{Cl}=3.934-5.109$, $P<0.001$ ) that of patients without drug dependence and without mental disorders, respectively. In the female population, suicide risk in insomnia patients was 4.186-fold (adjusted $\mathrm{HR}=4.186,95 \% \mathrm{Cl}=3.429-5.111, P<0.001$ ) that of patients without insomnia. Among patients aged 25-44 years, suicide risk in insomnia patients was 5.546-fold (adjusted HR=5. $546,95 \% \mathrm{Cl}=4.236-7.262, P<0.001)$ that of patients without insomnia. Furthermore, the suicide risk of insomnia patients with mental disorders was 18.322-fold that of patients without insomnia and mental disorders $(P<0.001)$.

Conclusion: Insomnia, low income, drug dependence, and mental disorders are independent risk factors for suicide attempts. Female patients and those aged 25-44 years are at high risk of suicide due to insomnia. Insomnia, mental disorders, and low income exhibit a synergistic effect on suicide attempts. Clinicians should pay attention to mental status and income level of insomnia patients.
\end{abstract}

Keywords: Insomnia, Suicide, National Health Insurance Research Database (NHIRD)

\footnotetext{
* Correspondence: chienwu@ndmctsgh.edu.tw

${ }^{1}$ National Defense Medical Center, School of Public Health 4325R, No. 161, Section 6, Min-Chuan East Road, Neihu District, Taipei City 11490, Taiwan, Republic of China

${ }^{2}$ National Defense Medical Center, Graduate Institute of Life Sciences 7115R,

No. 161, Section 6, Min-Chuan East Road, Neihu District, Taipei City 11490,

Taiwan, Republic of China

Full list of author information is available at the end of the article
}

(c) The Author(s). 2018 Open Access This article is distributed under the terms of the Creative Commons Attribution 4.0 International License (http://creativecommons.org/licenses/by/4.0/), which permits unrestricted use, distribution, and reproduction in any medium, provided you give appropriate credit to the original author(s) and the source, provide a link to the Creative Commons license, and indicate if changes were made. The Creative Commons Public Domain Dedication waiver (http://creativecommons.org/publicdomain/zero/1.0/) applies to the data made available in this article, unless otherwise stated. 


\section{Background}

Suicide is a serious public health problem around the world. The World Health Organization (WHO) reported that approximately 800,000 people worldwide die from suicide every year (an average of one death every $40 \mathrm{~s}$ ). In addition, suicide is the second leading cause of death among those aged 15-29 years globally, and the fifth leading cause of death among those aged 30-49 years [1]. Compared with death by suicide, there are many more suicide attempts every year [2], and according to a metaanalysis from Japan in 2008, a prior suicide attempt is the most important predictor of suicide [3]. Suicide, moreover, causes immense socioeconomic burdens on families, communities, and nations [4]. In 2016, the number of deaths from suicide in Taiwan was 3765 (a suicide death rate of 16.0 persons per 100,000 population), making suicide the twelfth leading cause of death in Taiwan. The suicide death rate among men was approximately 2.1-times higher than that among women (17.0 men and 7.7 women per 100,000 population). The suicide death rate in all age groups increased with age [5].

Insomnia is one of the most prevalent sleeping disorders in the world [6]. According to epidemiological studies around the world, the prevalence of insomnia in general populations is $10 \%-25 \%$ [7-10]. A 2017 report by the Taiwan Society of Sleep Medicine indicated that a tenth of the population across Taiwan suffers from chronic insomnia (prevalence rate of 11.3\%) [11]. Insomnia refers to difficulty falling asleep, remaining awake while trying to sleep, waking up often during the night, or still feeling tired after sleeping for a brief period, factors that subsequently influence daytime activities of daily living for more than four weeks. If insomnia persists for more than six months, it becomes a chronic condition influencing not only the person's psychology and physiology but also his or her health and activities of daily living (e.g., learning and working) [12].

Numerous studies have indicated that insomnia is associated with suicidal ideation [13-16], suicide attempts [17-19], and death by suicide [20-22] among adolescents and adults. These studies laid the foundation for the relationship between insomnia and suicide. However, there are some weakness in methodology and need future research to fill this gap. For example, some studies used a questionnaire asking a single question as criteria for determining insomnia or suicide [22]. Furthermore, mental disorders are a major confounding factor for insomnia and suicide, and over $90 \%$ of suicide decedents have at least one mental disorder [23]; however, some studies assessing the link between insomnia and suicide did not adjust for mental disorders [17, 20].

There are still different arguments about whether insomnia is an independent factor of suicide or if insomnia, as a symptom of mental disorders such as depression, increases the risk of suicide. Some studies have found that the relationship between insomnia and suicide is fully mediated by mental disorders [13, 14, 24]. Other studies have shown that insomnia remains an independent factor of suicidal ideation [15, 16], suicide attempt [19], and death by suicide [21, 22] after adjustments for mental disorders, substance abuse and alcohol abuse.

A meta-analysis by Pigeon et al. indicated that insomnia is still a predictor of suicide even after other factors have been adjusted for. However, the authors asserted that the samples collected are non-homogeneous, and samples for observational and clinical studies are confined to adolescents or adults only. Additionally, numerous studies are crosssectional studies [25], which cannot be used to elucidate whether insomnia increases suicide risk. Hence, we employed the National Health Insurance Research Database (NHIRD) in Taiwan to conduct a retrospective cohort study of whether insomnia increases the risk of suicide attempts.

\section{Methods \\ Data source}

Introduced in Taiwan in 1995, the NHRID contains the medical records of all insured. The database encompasses the information of more than $99 \%$ of the 23 million people who live in Taiwan; thus, the health care information contained therein represents evidence-based data in the medicine and healthcare sector [26]. This study analyzed the 2000-2013 inpatient expenditure files and the registry for contracted medical facilities in the NHRID.

\section{Design and sample}

The retrospective cohort study design method was adopted in this study. Inpatients aged 15 years or older who were newly diagnosed with insomnia (International.

Classification of Diseases, 9th Revision, Clinical Modifications [ICD-9-CM]: 307.41, 307.42, 780.52) were allocated to the study cohort. To ensure that the patients were newly diagnosed, we also excluded patients who were diagnosed with insomnia between 1997 and 1999. The study period began on January 1, 2000 and ended on December 31, 2013. In total, 159,989 participants were included. The average of follow-up for the diagnosis of insomnia was 6.47 years. To facilitate comparison of the study cohort, propensity score-matching based on sex, age group, and date of hospitalization was performed for a 1:2 pairing to identify the reference cohort ( $n=319,978$ inpatients who were diagnosed as not having insomnia). Furthermore, there was no difference in the cause of hospitalization at baseline (Additional file 1: Table S1).

\section{Outcome assessment}

All research participants were observed until the incidence of suicide attempts (ICD-9-CM E code: 950-959), loss to follow up, or until the end of December 31, 2013. 


\section{Covariates of interested}

The participants in this study were allocated into four groups by age: $15-24,25-44,45-64$, and $\geq 65$ years. Urbanization levels were categorized into high, moderate, and low levels of urbanization [27]. Low income included insured individuals in Category 5 (those to which laws and regulations governing social relief apply). Catastrophic illness was indicated in the copayment remarks of inpatient expenditure files. The Charlson comorbidity index (CCI) was used to weigh the 19 types of diseases by assigning each with a score of 1,2 , or 6 , and the scores were summed according to whether each patient had any of these diseases $[28,29]$. Because drug dependence, alcohol dependence, and mental disorders are risk factors for suicide attempts, drug dependence (ICD-9-CM 292, 304), alcohol dependence (ICD-9-CM 291, 303), and mental disorders (ICD-9CM 290-319, excluding 291, 292, 303, 304) were incorporated in the regression model for adjustments.

\section{Statistical analysis}

All analyses were performed using SPSS 22 (SPSS, Inc., Chicago, IL, U.S.). The $\chi^{2}$ test and Fisher exact test were used to compare the categorical variables of the two groups, and a t-test was conducted to compare the continuous variables of the two groups. With adjustments for sex, age, low income, catastrophic illness, urbanization, CCI, drug dependence, alcohol dependence, and mental disorders, Cox proportional hazards regression analyses were performed to assess the effects of insomnia on the risk of suicide attempts and presented as hazard ratio (HR) with a 95\% confidence interval (CI). Furthermore, differences in the risk of suicide attempts between the study and reference cohort were estimated using the Kaplan-Meier method with the log-rank test. A 2-tailed $p$-value $<0.05$ was considered to indicate statistical significance.

\section{Results}

Figure 1 shows the case-screening process (inclusion and exclusion) and follow-up results as well as the cumulative risk of suicide incidence between the two groups (patients with/without insomnia). Between 2000 and 2013, $16,716,547$ patients were hospitalized, 178,589 of which had insomnia. After exclusion criteria were applied (18,600 excluded), there were a total of 159,989 insomnia

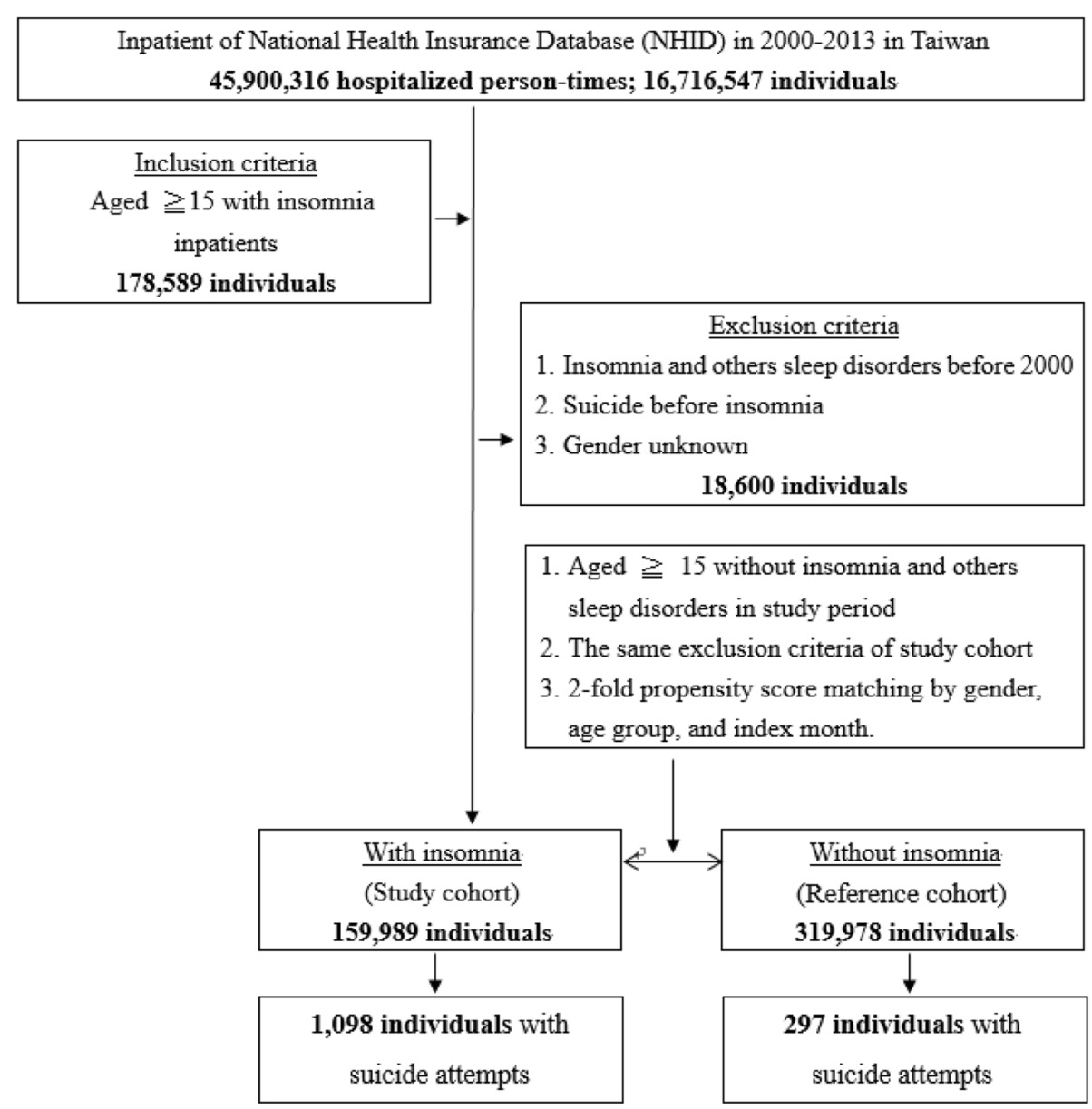

Fig. 1 Flowchart of study sample selection from the National Health Insurance Research Database in Taiwan 


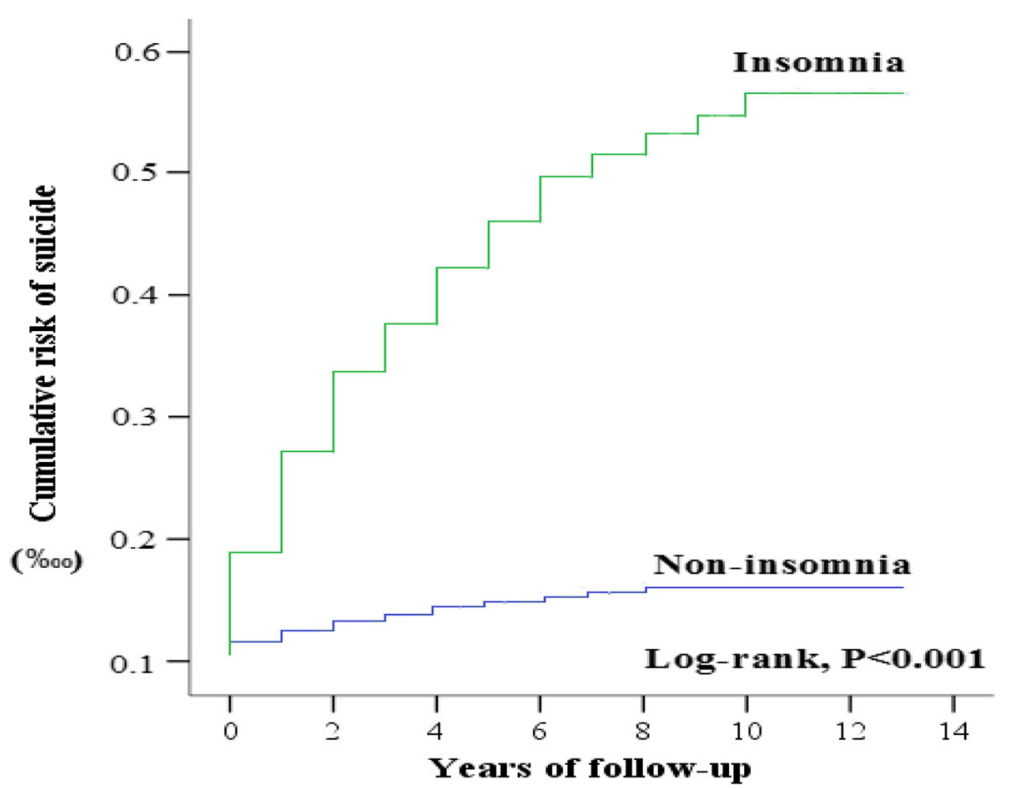

Fig. 2 Kaplan-Meier analysis of the cumulative risk of suicide attempts in 13 years of tracking stratified by insomnia with log-rank test

cases $(319,978$ patients were placed in the reference cohort based on 1:2 pairing) with a subsequent suicide incidence of $0.69 \%(1098 / 159,989)$, whereas the reference cohort exhibited a suicide incidence of $0.09 \%$ (297/ 319,978). The groups demonstrated significant difference (log-rank $P<0.001$ ). In other words, the probability (risk) of suicide attempts in insomnia patients was considerably higher than that in patients without insomnia. In addition, both groups exhibited a statistically significant difference after one year follow up (Fig. 2, Table 1). Furthermore, an average follow-up period for the diagnosis of insomnia to suicide attempts was 2.38 years.

Table 2 presents the basic characteristics of the 479,967 patients at the endpoint of the follow-up process (study cohort $=159,989$ insomnia patients; reference cohort = 319,978 non-insomnia patients). The study cohort exhibited a substantially higher incidence of suicide attempts compared with the reference cohort $(0.7 \%$ vs $0.1 \% ; P<0$. 001). The study cohort comprised significantly higher numbers of low income patients $(4.0 \%$ vs $1.5 \% ; P<0.001)$, patients with catastrophic illness $(31.2 \%$ vs $19.8 \%$; $P<0$. 001), and those who lived in working class urbanized townships $(33.4 \%$ vs $22.3 \% ; P<0.001)$ than the reference cohort. The numbers of patients with drug dependence (1. $0 \%$ vs $0.1 \% ; P<0.001)$, alcohol dependence $(3.5 \%$ vs $0.7 \%$; $p<0.001)$, and mental disorders (33.5\% vs $7.1 \% ; p<0.001)$ and other comorbidities in the study cohort were significantly higher than those of the reference cohort.

Table 3 shows the univariate and multivariate analysis results of the factors of suicide attempts. After the research variables (sex, age, low income, catastrophic illness, urbanization, CCI, drug dependence, alcohol dependence, and mental disorders) were adjusted for, the risk of suicide attempts among insomnia patients was 3.533 -fold that of non-insomnia patients $(P<0.001)$. The risk of suicide attempts among female patients was 1.522 -fold $(P<0.001)$ that of male patients. The risk of suicide attempts among patients aged 15-24, 25-44, 45-64 years was 6.000-fold, 3.581-fold, and 1.595-fold $(P<0.001)$ that of patients aged 65 years or older, respectively. The risk of suicide attempts among low-income patients and patients with catastrophic

Table 1 Kaplan-Meier analysis of the cumulative risk of suicide attempts in 13 years of tracking stratified by insomnia with log-rank test

\begin{tabular}{llll}
\hline Insomnia & $\begin{array}{l}\text { With } \\
(N=159,989)\end{array}$ & $\begin{array}{l}\text { Without } \\
(N=319,979)\end{array}$ & $P$-value \\
$\begin{array}{l}\text { X-year(s) of } \\
\text { suicide attempts }\end{array}$ & $\begin{array}{l}\text { Numbers of } \\
\text { suicide attempts }\end{array}$ & & \\
\hline 1 & 285 & 101 & $<0.001$ \\
2 & 525 & 157 & $<0.001$ \\
3 & 700 & 195 & $<0.001$ \\
4 & 808 & 222 & $<0.001$ \\
5 & 890 & 248 & $<0.001$ \\
6 & 965 & 265 & $<0.001$ \\
7 & 1020 & 276 & $<0.001$ \\
8 & 1052 & 286 & 0.001 \\
9 & 1070 & 295 & $<0.001$ \\
10 & 1084 & 296 & $<0.001$ \\
11 & 1198 & 297 & $<0.001$ \\
12 & 1198 & 297 & $<0.001$ \\
13 & 1198 & 297 & $<0.001$ \\
\hline
\end{tabular}


Table 2 Characteristics of study in the endpoint

\begin{tabular}{|c|c|c|c|c|c|c|c|}
\hline \multirow[b]{2}{*}{ Variables } & \multicolumn{2}{|l|}{ Total } & \multicolumn{2}{|c|}{ With insomnia } & \multicolumn{2}{|c|}{ Without insomnia } & \multirow[t]{2}{*}{$P$-value } \\
\hline & $n$ & $\%$ & $n$ & $\%$ & $n$ & $\%$ & \\
\hline Total & 479,967 & 100.0 & 159,989 & 33.3 & 319,978 & 66.7 & \\
\hline Suicide attempts & & & & & & & $<0.001$ \\
\hline with & 1395 & 0.3 & 1098 & 0.7 & 297 & 0.1 & \\
\hline without & 478,572 & 99.7 & 158,891 & 99.3 & 319,681 & 99.9 & \\
\hline Gender & & & & & & & 0.880 \\
\hline male & 235,771 & 49.1 & 78,615 & 49.1 & 157,156 & 49.1 & \\
\hline female & 244,196 & 50.9 & 81,374 & 50.9 & 162,822 & 50.9 & \\
\hline Age group (years) & & & & & & & 0.958 \\
\hline $15-24$ & 15,901 & 3.3 & 5330 & 3.3 & 10,571 & 3.3 & \\
\hline $25-44$ & 99,058 & 20.6 & 33,030 & 20.6 & 66,028 & 20.6 & \\
\hline $45-64$ & 172,650 & 36.0 & 57,504 & 35.9 & 115,146 & 36.0 & \\
\hline$\geqq 65$ & 192,358 & 40.1 & 64,125 & 40.1 & 128,233 & 40.1 & \\
\hline Low income & & & & & & & $<0.001$ \\
\hline yes & 11,251 & 2.3 & 6441 & 4.0 & 4810 & 1.5 & \\
\hline no & 468,716 & 97.7 & 153,548 & 96.0 & 315,168 & 98.5 & \\
\hline Catastrophic illness & & & & & & & $<0.001$ \\
\hline yes & 113,150 & 23.6 & 49,914 & 31.2 & 63,236 & 19.8 & \\
\hline no & 366,817 & 76.4 & 110,075 & 68.8 & 256,742 & 80.2 & \\
\hline Urbanization & & & & & & & $<0.001$ \\
\hline high & 150,124 & 31.3 & 41,562 & 26.0 & 108,562 & 33.9 & \\
\hline medium & 205,154 & 42.7 & 65,049 & 40.7 & 140,105 & 43.8 & \\
\hline low & 124,689 & 26.0 & 53,378 & 33.4 & 71,311 & 22.3 & \\
\hline $\mathrm{CCl}$ & $2.36 \pm 3.82$ & & $2.97 \pm 4.10$ & & $2.06 \pm 3.63$ & & $<0.001$ \\
\hline Drug dependence & & & & & & & $<0.001$ \\
\hline yes & 2051 & 0.4 & 1597 & 1.0 & 454 & 0.1 & \\
\hline no & 477,916 & 99.6 & 158,392 & 99.0 & 319,524 & 99.9 & \\
\hline Alcohol dependence & & & & & & & $<0.001$ \\
\hline yes & 7846 & 1.6 & 5674 & 3.5 & 2172 & 0.7 & \\
\hline no & 472,121 & 98.4 & 154,315 & 96.5 & 317,806 & 99.3 & \\
\hline Mental disorders & & & & & & & $<0.001$ \\
\hline yes & 76,322 & 15.9 & 53,537 & 33.5 & 22,785 & 7.1 & \\
\hline no & 403,645 & 84.1 & 106,452 & 66.5 & 297,193 & 92.9 & \\
\hline
\end{tabular}

$P$-value (category variable: Chi-square/Fisher exact test; continue variable: $t$-test)

illness was 1.434-fold and 1.286-fold that of their counterparts, respectively $(P<0.001)$. The risk of suicide attempts among patients with drug dependence and mental disorders was 1.592 -fold and 4.483-fold that of their counterparts, respectively $(P<0.001)$.

Table 4 shows the hierarchical analysis of various variables to elucidate the difference in risk of suicide attempts between insomnia and non-insomnia patients. The results indicated that (after other factors were adjusted for), in the female population, suicide risk in insomnia patients was 4.186-fold (adjusted $\mathrm{HR}=4.186$, 95\% CI $=3.429-5$. $111, P<0.001)$ that of patients without insomnia. Among patients aged 25-44 years, suicide risk in insomnia patients was 5.546-fold (adjusted $\mathrm{HR}=5.546,95 \% \mathrm{CI}=4$. $236-7.262, P<0.001)$ that of patients without insomnia.

Figures 3 and 4 show the interactive effects of insomnia, mental disorders, and low income after other factors were adjusted for. The risk of suicide attempts in insomnia patients without mental disorders was 4.960 -fold that of patients without insomnia and mental disorders. The risk of 
Table 3 Factors of suicide attempts by Cox proportional hazard model analysis

\begin{tabular}{|c|c|c|c|c|c|c|c|c|}
\hline \multirow{2}{*}{$\frac{\text { Variables }}{\text { Insomnia }}$} & \multirow[t]{2}{*}{ Crude HR } & \multicolumn{2}{|l|}{$95 \% \mathrm{Cl}$} & \multirow[t]{2}{*}{$P$-value } & \multirow[t]{2}{*}{ Adjusted HR } & \multicolumn{2}{|l|}{$95 \% \mathrm{Cl}$} & \multirow[t]{2}{*}{$P$-value } \\
\hline & & & & & & & & \\
\hline no & reference & & & & reference & & & \\
\hline yes & 7.416 & 6.524 & 8.431 & $<0.001$ & 3.533 & 3.059 & 4.080 & $<0.001$ \\
\hline \multicolumn{9}{|l|}{ Gender } \\
\hline female & reference & & & & reference & & & \\
\hline male & 1.289 & 1.159 & 1.433 & $<0.001$ & 1.522 & 1.361 & 1.702 & $<0.001$ \\
\hline \multicolumn{9}{|c|}{ Age group (years) } \\
\hline$\geqq 65$ & reference & & & & reference & & & \\
\hline $15-24$ & 6.477 & 5.292 & 7.928 & 0.001 & 6.000 & 4.876 & 7.383 & $<0.001$ \\
\hline $25-44$ & 4.909 & 4.238 & 5.687 & $<0.001$ & 3.581 & 3.069 & 4.177 & $<0.001$ \\
\hline $45-64$ & 1.747 & 1.487 & 2.052 & $<0.001$ & 1.595 & 1.357 & 1.876 & $<0.001$ \\
\hline \multicolumn{9}{|l|}{ Low income } \\
\hline no & reference & & & & reference & & & \\
\hline yes & 3.940 & 3.267 & 4.751 & $<0.001$ & 1.434 & 1.184 & 1.736 & $<0.001$ \\
\hline \multicolumn{9}{|c|}{ Catastrophic illness } \\
\hline No & reference & & & & reference & & & \\
\hline Yes & 1.972 & 1.771 & 2.196 & $<0.001$ & 1.286 & 1.143 & 1.446 & $<0.001$ \\
\hline \multicolumn{9}{|c|}{ Urbanization } \\
\hline high & reference & & & & reference & & & \\
\hline medium & 1.045 & 0.916 & 1.193 & 0.510 & 1.013 & 0.887 & 1.157 & 0.847 \\
\hline low & 1.569 & 1.372 & 1.795 & $<0.001$ & 1.073 & 0.937 & 1.230 & 0.309 \\
\hline $\mathrm{CCl}$ & 0.983 & 0.969 & 0.998 & 0.025 & 0.989 & 0.973 & 1.005 & 0.179 \\
\hline \multicolumn{9}{|c|}{ Drug dependence } \\
\hline no & reference & & & & reference & & & \\
\hline yes & 9.614 & 7.407 & 12.479 & $<0.001$ & 1.592 & 1.220 & 2.077 & 0.001 \\
\hline \multicolumn{9}{|c|}{ Alcohol dependence } \\
\hline no & reference & & & & reference & & & \\
\hline yes & 5.872 & 4.899 & 7.038 & $<0.001$ & 1.164 & 0.955 & 1.420 & 0.133 \\
\hline \multicolumn{9}{|c|}{ Mental disorders } \\
\hline no & reference & & & & reference & & & \\
\hline yes & 9.742 & 8.723 & 10.879 & $<0.001$ & 4.483 & 3.934 & 5.109 & $<0.001$ \\
\hline
\end{tabular}

suicide attempts in non-insomnia patients with mental disorders was 8.237 -fold that of patients without insomnia and mental disorders. The risk of suicide attempts in insomnia patients with mental disorders was 18.322-fold that of patients without insomnia and mental disorders $(P$ $<0.001$ ) (Fig. 3). The risk of suicide attempts in non-lowincome patients with insomnia was 3.521-fold that of non-insomnia patients who were not in the low-income group. The risk of suicide attempts in low-income patients without insomnia was 1.330 -fold that of non-insomnia patients who were not in the low-income group. The risk of suicide attempts in low-income patients with insomnia was 5.084-fold that of non-insomnia patients who were not in the low-income group $(P<0.001)$ (Fig. 4$)$.

\section{Discussion}

Our study found that insomnia remained an independent risk factor for suicide attempts after adjustments for mental disorders, alcohol dependence, and drug dependence. To the best of our knowledge, our study is the first retrospective cohort study to use population-based data and clinical diagnosis as the criteria for determining insomnia and suicide. The findings can reinforce the deficiencies of other relevant studies.

In recent years, some studies found that mental disorders, alcohol abuse, and drug abuse do not fully mediate the association between insomnia and suicide. A study conducted in 2012 regarding members of the military in the United States reported that insomnia was an independent risk 
Table 4 Factors of suicide attempts stratified by variables listed in the table by Cox proportional hazard model analysis

\begin{tabular}{|c|c|c|c|c|c|c|c|c|c|c|c|}
\hline \multirow[t]{2}{*}{ Variables } & \multicolumn{3}{|c|}{ With insomnia } & \multicolumn{3}{|c|}{ Without insomnia } & \multirow[t]{2}{*}{ Ratio } & \multirow{2}{*}{$\begin{array}{l}\text { Adjusted } \\
\text { HR }\end{array}$} & \multirow[t]{2}{*}{$95 \% \mathrm{Cl}$} & & \multirow[t]{2}{*}{$P$-value } \\
\hline & Event & PYs & Rate (per $10^{3} \mathrm{PYs}$ ) & Event & PYS & Rate (per $10^{3} \mathrm{PYs}$ ) & & & & & \\
\hline Total & 1098 & 953,594 & 115.143 & 297 & $1,913,519$ & 15.521 & 7.418 & 3.533 & 3.059 & 4.080 & $<0.001$ \\
\hline \multicolumn{12}{|l|}{ Gender } \\
\hline female & 633 & 468,954 & 134.981 & 153 & 941,910 & 16.244 & 8.310 & 4.186 & 3.429 & 5.111 & $<0.001$ \\
\hline male & 465 & 484,640 & 95.948 & 144 & 971,609 & 14.821 & 6.474 & 2.861 & 2.323 & 3.523 & $<0.001$ \\
\hline \multicolumn{12}{|c|}{ Age group (years) } \\
\hline $15-24$ & 119 & 42,625 & 279.179 & 33 & 85,435 & 38.626 & 7.228 & 2.778 & 1.747 & 4.417 & $<0.001$ \\
\hline $25-44$ & 546 & 198,523 & 275.031 & 80 & 400,512 & 19.974 & 13.769 & 5.546 & 4.236 & 7.262 & $<0.001$ \\
\hline $45-64$ & 264 & 317,825 & 83.065 & 105 & 637,466 & 16.471 & 5.043 & 2.637 & 2.053 & 3.389 & $<0.001$ \\
\hline$\geqq 65$ & 169 & 394,621 & 42.826 & 79 & 790,106 & 9.999 & 4.283 & 2.900 & 2.188 & 3.844 & $<0.001$ \\
\hline \multicolumn{12}{|l|}{ Low income } \\
\hline no & 987 & 915,626 & 107.795 & 288 & $1,884,404$ & 15.283 & 7.053 & 3.487 & 3.009 & 4.040 & $<0.001$ \\
\hline yes & 111 & 37,968 & 292.351 & 9 & 29,115 & 30.912 & 9.458 & 4.069 & 2.012 & 8.228 & $<0.001$ \\
\hline \multicolumn{12}{|c|}{ Catastrophic illness } \\
\hline no & 635 & 645,939 & 98.306 & 220 & $1,504,259$ & 14.625 & 6.722 & 3.769 & 3.171 & 4.479 & $<0.001$ \\
\hline yes & 463 & 307,655 & 150.493 & 77 & 409,260 & 18.814 & 7.999 & 2.916 & 2.255 & 3.770 & $<0.001$ \\
\hline \multicolumn{12}{|l|}{ Urbanization } \\
\hline high & 291 & 243,831 & 119.345 & 84 & 661,398 & 12.700 & 9.397 & 3.911 & 2.964 & 5.161 & $<0.001$ \\
\hline medium & 398 & 369,572 & 107.692 & 132 & 835,185 & 15.805 & 6.814 & 3.320 & 2.655 & 4.153 & $<0.001$ \\
\hline low & 409 & 340,191 & 120.227 & 81 & 416,936 & 19.427 & 6.188 & 3.355 & 2.592 & 4.342 & $<0.001$ \\
\hline \multicolumn{12}{|c|}{ Drug dependence } \\
\hline no & 1044 & 942,877 & 110.725 & 292 & $1,910,198$ & 15.286 & 7.243 & 3.569 & 3.086 & 4.127 & $<0.001$ \\
\hline yes & 54 & 10,717 & 503.872 & 5 & 3321 & 150.557 & 3.347 & 2.079 & 0.817 & 5.286 & 0.207 \\
\hline \multicolumn{12}{|c|}{ Alcohol dependence } \\
\hline no & 978 & 916,596 & 106.699 & 288 & $1,899,319$ & 15.163 & 7.037 & 3.513 & 3.032 & 4.072 & $<0.001$ \\
\hline yes & 120 & 36,998 & 324.342 & 9 & 14,200 & 63.380 & 5.117 & 3.844 & 1.936 & 7.634 & $<0.001$ \\
\hline \multicolumn{12}{|c|}{ Mental disorders } \\
\hline no & 300 & 618,043 & 48.540 & 181 & $1,763,589$ & 10.263 & 4.730 & 4.798 & 3.968 & 5.801 & $<0.001$ \\
\hline yes & 798 & 335,551 & 237.818 & 116 & 149,930 & 77.369 & 3.074 & 2.223 & 1.823 & 2.711 & $<0.001$ \\
\hline
\end{tabular}

factor for suicidal ideation after adjustments for depression, hopelessness, post-traumatic stress disorder, anxiety, drug abuse, and alcohol abuse. However, there were no significant associations between insomnia and suicide attempts [15]. Different from the above study [15] using a 3-item questionnaire as the criteria for insomnia, our study used the clinical diagnosis as the criteria for determining insomnia. Another study conducted in the United States in 2016 revealed that insomnia symptoms increase the risk of suicidal ideation and attempts in adolescents with adjustments for mental disorders and substance use disorders [19]. However, the above study [19] had several limitations. First, the study was cross-sectional. No temporal relationships could be established. Second, all measures were based on self-report, thus the data were subject to response and recall bias. Therefore, based on above study [19], we established insomnia as an independent predictor of suicide attempts.

Our study found that insomnia and mental disorders have a synergistic effect on the risk of suicide attempts. The risk of suicide attempts in insomnia patients with mental disorders was 18-fold that of patients without insomnia and mental disorders. However, no comparison with past studies can be made because investigations concerning the interactive effects of insomnia and mental disorders on suicide attempts are lacking. A cross-sectional study conducted in 2018 in the United States reported that poor sleep quality will increase the risk of suicide after adjusting for depression among adolescents. However, sleep problems and depression do not interact with the risk of suicide [30]. Different from above study [30] focus on the association between sleep problems and suicide, our study 


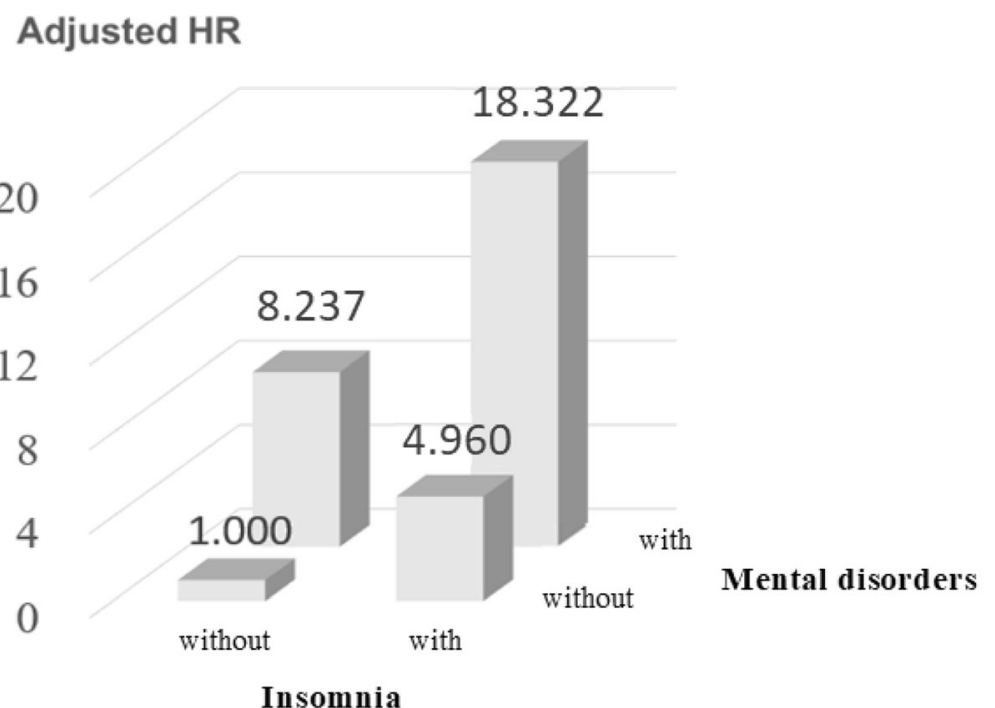

Fig. 3 Interaction for risk of suicide attempts by insomnia and mental disorders

focused on insomnia and suicide attempts. Therefore, it cannot be compared.

The mechanisms underlying the relationship between insomnia and suicide remain unclear. In 2013, a systematic literature review of adult insomnia and suicide conducted in the United States indicated that the mechanisms of insomnia and suicide involve both physiological and psychological dimensions [31]. The physiological mechanism includes a reduction in serotonin [32] and hypothalamic-pituitary-adrenal (HPA) axis dysfunction [33]. The psychological mechanism is associated with dysfunctional beliefs and attitudes about sleep (DBAS) [15] and depression [34]. Furthermore, a previous study found that fatigue resulting from sleep disorders may lead to hopelessness and decrease impulse control, both demonstrated risk factors for suicide [35]. The above possible mechanisms between insomnia and suicide require further studies for confirmation.

Our study determined that the risk of suicide attempts in patients with drug dependence was 1.592 -fold that of patients without drug dependence. Several studies have indicated that the abuse of drugs such as marijuana [36], heroin [37, 38], and nicotine [39] are risk factors for suicide. A review article reported that $25 \%$ to $50 \%$ of people who

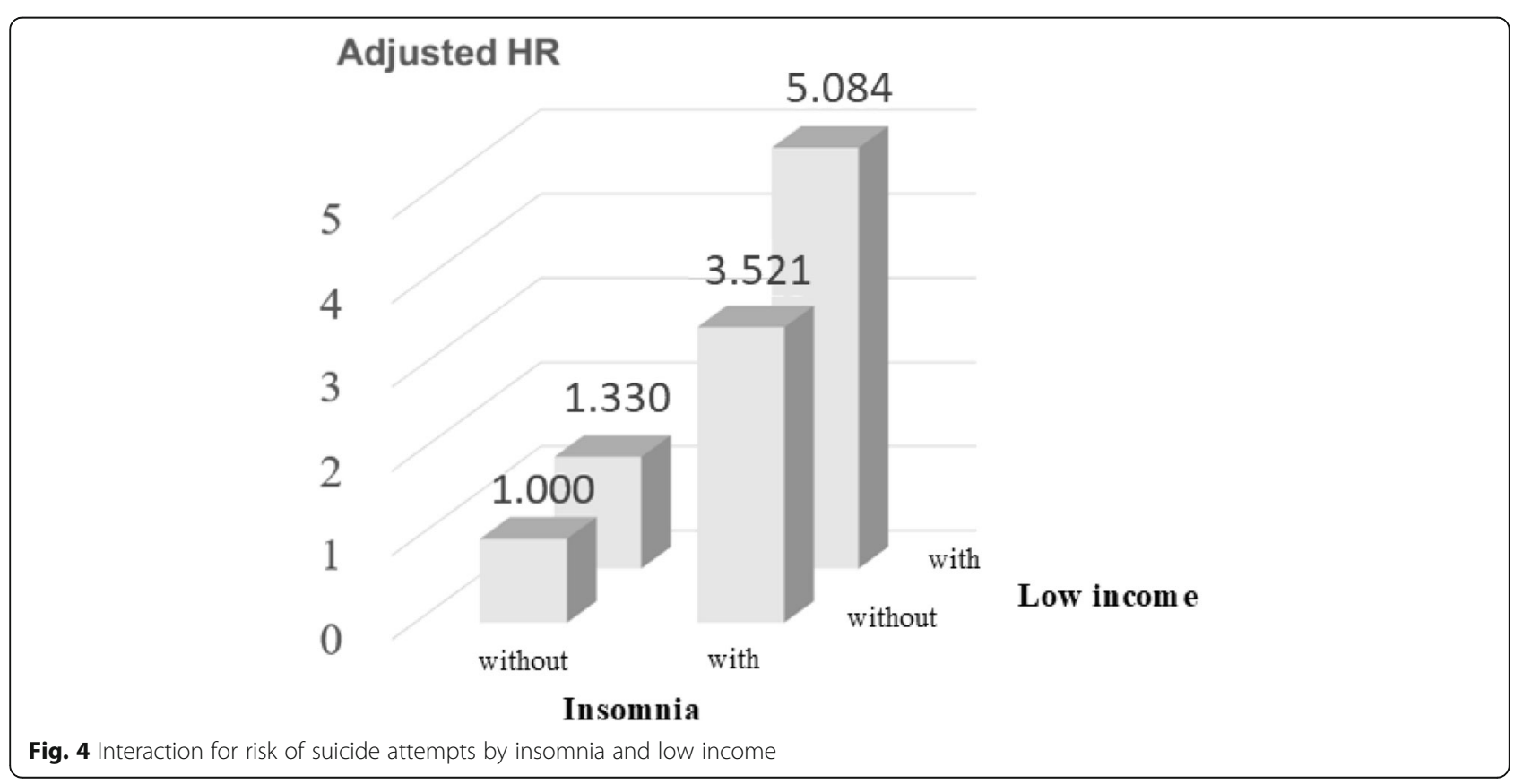


are suicidal are dependent on alcohol or drugs, and the risk of suicide increases if these people have mental disorders [40]. A study conducted in 1995 regarding indigenous peoples in Taiwan reported that the risk of suicide in people with substance dependence and depression was 470.2-fold that of people without substance dependence and depression [41]. In our study, we found that the risk of suicide attempts in patients with both substance dependence and depression was 22.7-fold that of their counterparts who did not fact these issues, which is lower than the finding of the aforementioned study. This difference might be attributable to social group, population, and cultural discrepancies. Another study conducted in the United States in 1993 revealed that the risk of suicide in patients with substance dependence and an emotional disorder was 17.0-fold that of their counterparts [42]. By contrast, we determined that the risk of suicide attempts in patients with both substance dependence and emotional disorder was 23.4-fold that of their counterparts. Similar to the Taiwan study [41], the focus of substance abuse in this study was alcohol and drugs; however, we reported a lower rate of substance abuse (alcohol abuse $=9.2 \%$ and drug abuse $=4.3 \%$ ) compared with that of [41] (alcohol abuse $=24.3 \%$ and drug abuse $=13$. $4 \%)$. This difference is possibly attributed to the more stringent standard of substance abuse (individuals exhibiting substance dependence) adopted in the present study.

Our study found that the risk of suicide attempts in patients with mental disorders was 4.483 -fold that of patients without mental disorders. In western countries such as the United Kingdom, a study reported that $90 \%$ of suicides were associated with a history of mental disorders [43]. In Asian countries such as China, $40 \%$ to $70 \%$ of suicides were associated with a history of mental disorders [44, 45]. Multiple studies have indicated that different types of mental disorders predict different levels of suicide risk [46-49]. In particular, schizophrenia has the highest risk for suicide [50]. In our study, depression had the highest risk for suicide attempts, followed by schizophrenia. Another study revealed that the risk of suicide in patients with more than one mental disorders was 1.5-2.5-fold that of patients with only one type of mental disorders [43]. We incorporated depression, anxiety, bipolar disorder, and schizophrenia for further analysis and found that for every increase in the number of mental disorders, the risk of suicide attempts increased by 0.6 -fold. This finding is somewhat similar to that reported in the aforementioned study.

In 2016, a United Kingdom systematic literature review indicated that low income, low socioeconomic status, and unemployment predicted higher levels of suicidal ideation, suicidal behavior, and death by suicide [51]. Our study found that risk of suicide attempts in low-income patients was 1.434-fold that of their counterparts (adjusted HR $=1$. $431,95 \% \mathrm{CI}=1.182-1.732, P<0.001)$. Furthermore, the risk of suicide attempts in insomnia patients who earn low levels of income was 4.960 -fold that of their counterparts $(P<0.001)$. This result implies that under the interactive effects of an economic stressor (low income) and insomnia, suicide became a major option for this particular population (particularly women, people aged 25-44 years, and breadwinners in the family), thereby leading to regrettable circumstances.

Our study has the following limitations. First, due to the limited types of data provided in the NHIRD, we were unable to acquire important suicide-related information (e.g., stigma, family history of suicide, occurrence of material events, and network of support). Second, our study adopted inpatient expenditure files from the NHIRD for analysis; therefore, our study findings may not be generalizable to the entire population without including outpatient cases. Third, all information was collected from medical record (NHIRD). The possibility of information bias cannot be ruled out.

\section{Conclusions}

Insomnia is a risk factor for suicide attempts and, indeed, increases the risk for suicide. In addition, low income, drug dependence, and mental disorders are risk factors for suicide attempts. Female patients and those aged 25-44 years are high-risk groups. Risk of suicide attempts is higher in low-income individuals with insomnia and mental disorders. Clinicians should pay attention to the mental status and income level of insomnia patients and implement early suicide prevention intervention. If members of the general public have friends who suffer from insomnia, they should pay attention to the mental and financial status of their friends in order to reduce the probability of suicide.

\section{Additional file}

Additional file 1: Table S1. Main diagnosis for hospitalization in the baseline. (DOCX 15 kb)

\section{Abbreviations \\ CCl: The Charlson comorbidity index; Cl: Confidence interval; HR: Hazard ratio; ICD-9-CM: The International Classification of Diseases, 9th Revision, Clinical Modifications; NHIRD: National Health Insurance Research Database; WHO: World Health Organization}

\section{Acknowledgments}

This study was supported by grants from Tri-service Hospital Research Foundation (TSGH-C107-004), and the sponsor has no role in study design, data collection and analysis, decision to publish, or preparation of the manuscript.

\section{Availability of data and materials}

Data are from the National Health Institute Research Database (NHIRD), which are available to researchers in Taiwan and have been extensively used in epidemiologic studies. It is permissible to use these data for academic purposes only after providing proof to National Health Research Institute. Thus, the data cannot be made publicly available. Data requests may be sent to National Health Institute Research Database (http://nhird.nhri.org.tw/) at nhird@nhri.org.tw. 


\section{Authors' contributions}

$\mathrm{CCH}, \mathrm{LCH}$ and $\mathrm{CWC}$ contributed to the study design. Data analyses and interpretation were performed by LHT, PHJ, WCC, CWL and CWC. LHT, LCH and CWC drafted and revised the manuscript. All authors read and approved the final manuscript.

\section{Ethics approval and consent to participate}

The study protocol was approved by the approval institutional review board, Tri-Service General Hospital. (TSGHIRB No. 1-106-05-169).

\section{Competing interests}

The authors declare that they have no competing interests.

\section{Publisher's Note}

Springer Nature remains neutral with regard to jurisdictional claims in published maps and institutional affiliations.

\section{Author details}

'National Defense Medical Center, School of Public Health 4325R, No. 161, Section 6, Min-Chuan East Road, Neihu District, Taipei City 11490, Taiwan, Republic of China. ${ }^{2}$ National Defense Medical Center, Graduate Institute of Life Sciences 7115R, No. 161, Section 6, Min-Chuan East Road, Neihu District, Taipei City 11490, Taiwan, Republic of China. ${ }^{3}$ Department of Family and Community Medicine, National Defense Medical Center, Tri-Service General Hospital Taipei, No. 325, Section 2, Cheng-Kung Road, Taipei City 11490 Taiwan, Republic of China. ${ }^{4}$ Department of Medical Research 7115R, National Defense Medical Center, Tri-Service General Hospital Taipei, No. 325, Section 2, Cheng-Kung Road, Taipei City 11490, Taiwan, Republic of China.

\section{Received: 7 January 2018 Accepted: 23 April 2018}

\section{Published online: 02 May 2018}

\section{References}

1. World Health Organization. Suicide: Fact sheet. 2017 [http://www.who.int/ mediacentre/factsheets/fs398/en/].

2. Nock MK, Borges G, Bromet EJ, Cha CB, Kessler RC, Lee S. Suicide and suicidal behavior. Epidemiol Rev. 2008;30:133-54.

3. Yoshimasu K, Kiyohara C, Miyashita K. Stress research Group of the Japanese Society for hygiene. Suicidal risk factors and completed suicide: metaanalyses based on psychological autopsy studies. Environ Health Prev Med. 2008;13(5):243-56.

4. World Health Organization. Preventing suicide: A global imperative. 2014 [http/// www.who.int/mental_health/suicide-prevention/world_report_2014/en/].

5. Ministry of Health and Welfare, Taiwan. 2016 statistical results on causes of death in Taiwan. 2016 [https://www.mohw.gov.tw/cp-16-33598-1.html].

6. Unbehaun T, Spiegelhalder K, Hirscher V, Riemann D. Management of insomnia: update and new approaches. Nat Sci Sleep. 2010;28(2):127-38.

7. Doghramji K. The epidemiology and diagnosis of insomnia. Am J Manag Care. 2006;12(Suppl 8):S214-20

8. Cho YW, Shin WC, Yun CH, Hong SB, Kim J, Earley CJ. Epidemiology of insomnia in korean adults: prevalence and associated factors. I Clin Neurol. 2009;5(1):20-3.

9. Chan-Chee C, Bayon V, Bloch J, Beck F, Giordanella JP, Leger D. Epidemiology of insomnia in France. Rev Epidemiol Sante Publique. 2011; 59(6):409-22.

10. Ikeda M, Kaneita Y. The newest epidemiology trend of insomnia. Nihon Rinsho. 2014;72(3):573-7.

11. Taiwan Society of Sleep Medicine. 2017 trend in prevalence of sleep problems in Taiwan: a 10-years cross sectional study. 2017 [http://www. tssm.org.tw/file/1494489550.pdf].

12. Chung WS, Li FC, Ho FM. Classification and Treatment of insomnia. Taiwan Med J. 2013;56(12):642-5

13. Nadorff MR, Nazem S, Fiske A. Insomnia symptoms, nightmares, and suicidal ideation in a college student sample. Sleep. 2011;34(1):93-8.

14. Nadorff MR, Fiske A, Sperry JA, Petts R, Gregg JJ. Insomnia symptoms, nightmares, and suicidal ideation in older adults. J Gerontol B Psychol Sci Soc Sci. 2013;68(2):145-52.

15. Ribeiro JD, Pease JL, Gutierrez PM, Silva C, Bernert RA, Rudd MD, et al. Sleep problems outperform depression and hopelessness as cross-sectional and longitudinal predictors of suicidal ideation and behavior in young adults in the military. J Affect Disord. 2012;136(3):743-50.
16. Richardson JD, Thompson A, King L, Corbett B, Shnaider P, St Cyr K, et al. Insomnia, psychiatric disorders and suicidal ideation in a National Representative Sample of active Canadian forces members. BMC Psychiatry. 2017:17(1):211.

17. Hall RC, Platt DE, Hall RC. Suicide risk assessment: a review of risk factors for suicide in 100 patients who made severe suicide attempts. Evaluation of suicide risk in a time of managed care. Psychosomatics. 1999;40(1):18-27.

18. Kay DB, Dombrovski AY, Buysse DJ, Reynolds CF, Begley A, Szanto K. Insomnia is associated with suicide attempt in middle-aged and older adults with depression. Int Psychogeriatr. 2016;28(4):613-9.

19. Wong MM, Brower KJ, Craun EA. Insomnia symptoms and suicidality in the National Comorbidity Survey - adolescent supplement. J Psychiatr Res. 2016; 81:1-8.

20. Fujino Y, Mizoue T, Tokui N, Yoshimura T. Prospective cohort study of stress, life satisfaction, self-rated health, insomnia, and suicide death in Japan. Suicide Life Threat Behav. 2005;35(2):227-37.

21. Goldstein TR, Bridge JA, Brent DA. Sleep disturbance preceding completed suicide in adolescents. J Consult Clin Psychol. 2008;76(1):84-91.

22. Bjorngaard JH, Bjerkeset O, Romundstad P, Gunnell D. Sleeping problems and suicide in 75,000 Norwegian adults: a 20 year follow-up of the HUNT I study. Sleep. 2011;34(9):1155-9.

23. Bertolote JM, Fleischmann A, De Leo D, Wasserman D. Psychiatric diagnoses and suicide: revisiting the evidence. Crisis. 2004;25(4):147-55.

24. Bernert RA, Joiner TE, Cukrowicz KC, Schmidt NB, Krakow B. Suicidality and sleep disturbances. Sleep. 2005;28(9):1135-41.

25. Pigeon WR, Pinquart M, Conner K. Meta-analysis of sleep disturbance and suicidal thoughts and behaviors. J Clin Psychiatry. 2012;73(9):e1160-7.

26. National Health Research Institutes, Taiwan. The National Health Insurance Research Database: background. 2003 [https://nhird.nhri.org.tw/en/index. html].

27. Liu CY, Hung YT, Chuang YL, Chen YJ, Weng WS, Liu JS, et al. Incorporating development stratification of Taiwan townships into sampling Design of Large Scale Health Interview Survey. J Health Manag. 2006;4(1):1-22.

28. Charlson ME, Pompei P, Ales KL, Mackenzie CR. A new method of classifying prognostic comorbidity in longitudinal studies: development and validation. J Chronic Dis. 1987:40(5):373-83.

29. Deyo RA, Cherkin DC, Ciol MA. Adapting a clinical comorbidity index for use with ICD-9-CM administrative databases. J Clin Epidemiol. 1992:45(6):613-9.

30. Becker SP, Dvorsky MR, Holdaway AS, Luebbe AM. Sleep problems and suicidal behaviors in college students. J Psychiatr Res. 2018;99:122-8.

31. McCall WV, Black CG. The link between suicide and insomnia: theoretical mechanisms. Curr Psychiatry Rep. 2013;15(9):389.

32. Elmenhorst D, Kroll T, Matusch A, Bauer A. Sleep deprivation increases cerebral serotonin 2A receptor binding in humans. Sleep. 2012;35(12):1615-23.

33. Bonnet MH, Arand DL. 24-hour metabolic rate in insomniacs and matched normal sleepers. Sleep. 1995;18(7):581-8.

34. Kitamura S, Hida A, Watanabe M, Enomoto M, Aritake-Okada S, Moriguchi Y, et al. Evening preference is related to the incidence of depressive states independent of sleep-wake conditions. Chronobiol Int. 2010;27(9-10):1797-812.

35. Joiner TE, Brown JS, Wingate LR. The psychology and neurobiology of suicidal behavior. Annu Rev Psychol. 2005;56:287-314.

36. Borges G, Benjet C, Orozco R, Medina-Mora ME, Menendez D. Alcohol, cannabis and other drugs and subsequent suicide ideation and attempt among young Mexicans. J Psychiatr Res. 2017;91:74-82.

37. Kazour F, Soufia M, Rohayem J, Richa S. Suicide risk of heroin dependent subjects in Lebanon. Community Ment Health J. 2016;52(5):589-96.

38. Darke S, Ross J, Marel C, Mills KL, Slade T, Burns L, et al. Patterns and correlates of attempted suicide amongst heroin users: 11-year follow-up of the Australian treatment outcome study cohort. Psychiatry Res. 2015;227(23):166-70.

39. Bohnert KM, llgen MA, McCarthy JF, Ignacio RV, Blow FC, Katz IR. Tobacco use disorder and the risk of suicide mortality. Addiction. 2014;109(1):155-62.

40. Schneider B. Substance use disorders and risk for completed suicide. Arch Suicide Res. 2009:13(4):303-16.

41. Cheng AT. Mental illness and suicide. A case-control study in East Taiwan. Arch Gen Psychiatry. 1995;52(7):594-603.

42. Brent DA, Perper JA, Moritz G, Allman C, Friend A, Roth C, et al. Psychiatric risk factors for adolescent suicide: a case-control study. J Am Acad Child Adolesc Psychiatry. 1993;32(3):521-9.

43. Cavanagh JT, Carson AJ, Sharpe M, Lawrie SM. Psychological autopsy studies of suicide: a systematic review. Psychol Med. 2003;33(3):395-405. 
44. Zhang J, Xiao S, Zhou L. Mental disorders and suicide among young rural Chinese: a case-control psychological autopsy study. Am J Psychiatry. 2010; 167(7):773-81.

45. Jia $C X$, Wang $L L, X u A Q$, Dai $A Y$, Qin P. Physical illness and suicide risk in rural residents of contemporary China: a psychological autopsy case-control study. Crisis. 2014;35(5):330-7.

46. Indu PS, Anilkumar TV, Pisharody R, Russell PSS, Raju D, Sarma PS, et al. Prevalence of depression and past suicide attempt in primary care. Asian $J$ Psychiatr. 2017;27:48-52.

47. Thibodeau MA, Welch PG, Sareen J, Asmundson GJ. Anxiety disorders are independently associated with suicide ideation and attempts: propensity score matching in two epidemiological samples. Depress Anxiety. 2013; 30(10):947-54

48. McGrady A, Lynch D, Rapport D. Psychosocial factors and comorbidity associated with suicide attempts: findings in patients with bipolar disorder. Psychopathology. 2017;50(2):171-4.

49. Jakhar K, Beniwal RP, Bhatia T, Deshpande SN. Self-harm and suicide attempts in schizophrenia. Asian J Psychiatr. 2017;30:102-6.

50. Limosin F, Loze JY, Philippe A, Casadebaig F, Rouillon F. Ten-year prospective follow-up study of the mortality by suicide in schizophrenic patients. Schizophr Res. 2007;94(1-3):23-8.

51. lemmi V, Bantjes J, Coast E, Channer K, Leone T, McDaid D, et al. Suicide and poverty in low-income and middle-income countries: a systematic review. Lancet Psychiatry. 2016;3(8):774-83.

Ready to submit your research? Choose BMC and benefit from:

- fast, convenient online submission

- thorough peer review by experienced researchers in your field

- rapid publication on acceptance

- support for research data, including large and complex data types

- gold Open Access which fosters wider collaboration and increased citations

- maximum visibility for your research: over $100 \mathrm{M}$ website views per year

At BMC, research is always in progress.

Learn more biomedcentral.com/submissions 